Artículos Originales / Original Articles

\title{
Reproducibilidad de la escala de Masmali en la valoración de la película lagrimal
}

\author{
Reproducibility of the Masmali scale in the assessment of the lagrimal film
}

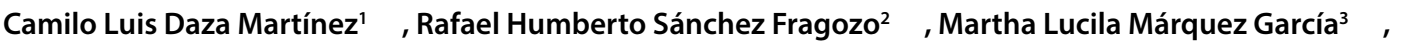 \\ Juan José Barrios Arlante ${ }^{4}$ (D)
}

Citación: Daza C, Sánchez-Fragozo RH, Márquez M, Barrios-Arlante JJ. Reproducibilidad de la escala de Masmali en la valoración de la película
lagrimal. Ustasalud 2022;21(1): 9-16. DOI:https://doi.org/10.15332/us.v21i1.2585

Licencia Creative Commons

(c) (1) (3) $\odot$ alterarlos, adicionalmente se debe reconocer la autoría de las personas que figuran en las publicaciones, pero estas no podrán ser comercializadas.

\section{Resumen}

Objetivo: determinar la reproducibilidad inter evaluador de la escala de Masmali en función del test de Ferning para la evaluación de la película lagrimal. Materiales y Métodos: se realizó un estudio observacional analítico de evaluación de pruebas diagnósticas. La muestra estuvo integrada por 50 estudiantes del programa de Optometría de la Universidad Santo Tomás, Bucaramanga. Se evaluó la osmolaridad de la película lagrimal de los individuos a través del test de Ferning. Dos observadores clasificaron los patrones en helecho obtenidos, empleando la escala de Masmali para determinar su reproducibilidad. Resultados: se demostró que la escala de Masmali es una herramienta de medición de la osmolaridad lagrimal válida; sus resultados son confiables y reproducibles. La concordancia interevaluador fue moderada con un Kappa Ponderado de 0,5658 con un 88\% de acuerdo y un error estándar de Kappa de 0,0995 y un valor $p$ de $<0,0001$. Conclusiones: se recomienda el uso de la escala de Masmali como un método de evaluación del fluido lagrimal, por arrojar resultados confiables. No obstante, estos deben ser correlacionados con otras pruebas de superficie ocular para la emisión de diagnósticos en la evaluación de las disfunciones lagrimales.

Palabras clave: Test de Ferning, escala de Masmali, lágrima, ojo seco, osmolaridad lagrimal.

\section{Abstract}

Objective: To determine the inter-rater reproducibility of the Masmali scale based on the Ferning Test for the evaluation of tear film. Materials and methods: An analytical observational study was carried out to evaluate diagnostic tests. The sample consisted of 50 students from the optometry program of the Universidad Santo Tomás, Bucaramanga. The osmolarity of the tear film of the individuals was evaluated through the Ferning test. Two observers classified the fern patterns obtained, using the Masmali scale to determine their reproducibility. Results: the Masmali scale was shown to be a valid tear osmolarity measurement tool; its results are reliable and reproducible. The inter-rater agreement was moderate with a Weighted Kappa of 0.5658 with $88 \%$ agreement and a standard error of kappa of 0.0995 and a $p$ value of $<0.0001$. Conclusions: The Masmali scale based on the Ferning Test proved to be reproducible and reliable considering the results obtained.

Keywords: Ferning test, Masmali scale, tear, dry eye, tear osmolarity.
1 Optómetra. Especialista en Segmento Anterior y Lentes de Contacto, Universidad Santo Tomás Seccional Bucaramanga. Colombia.

2 Optómetra. Especialista en Segmento Anterior y Lentes de Contacto, Universidad Santo Tomás Seccional Bucaramanga. Colombia.

3 Optómetra. Especialista en Segmento Anterior y Lentes de Contacto, Universidad Santo Tomás Seccional Bucaramanga. Colombia.

4 Magíster en Física, doctor en educación, grupo Interdisciplinario de Investigaciones Epidemiológicas en el Sistema Visual (GIESVI). Universidad Santo Tomás Seccional Bucaramanga. Colombia.

Autor de correspondencia:

Camilo Luis Daza Martínez

Correo electrónico:

camilo.daza01@ustabuca.edu.co
Recibido para publicación: 5 de octubre de 2020

Aceptado para publicación:

30 de junio de 2021 


\section{INTRODUCCIÓN}

Una de las funciones de la película lagrimal es la preservación de la fisiología del segmento anterior ocular, proporcionando estabilidad a su superficie. Esta aporta nutrientes, hidratación, protección antimicrobiana, favorece la eliminación de detritus y aporta a la óptica ocular [1].

El sistema lagrimal está conformado por la glándula lagrimal principal, la cual se encarga de producir la porción de lágrima refleja y las glándulas accesorias (Wolfring, Manz y Krause) que se hallan principalmente en los fondos de saco conjuntivales; estas tienen a cargo la secreción acuosa de lágrima basal. En la conjuntiva existen además numerosas células caliciformes que contribuyen también a la formación de este fluido, especialmente a la porción mucinosa del mismo [2]. Adicionalmente, las glándulas de Meibomio, ubicadas en el tarso palpebral, hacen su aporte, secretando una capa lipídica desde el borde libre de los párpados. Esta se ubica superficialmente y su función es evitar la evaporación de la porción acusa [3].

El espesor de la película lagrimal es aproximadamente de 7,1 micras. El componente externo lipídico mide en condiciones normales, 0,1 micras y la porción acuosa, cerca de 7 micras; este contiene un gradiente de mucinas, las cuales van disminuyendo en concentración desde la parte más interna a la capa lipídica exterior. La mucina se encarga de proporcionar un recubrimiento hidrofílico a la superficie de la córnea, para permitir un esparcimiento lagrimal uniforme [3]. Todos estos componentes deben permanecer en equilibrio, pues la alteración de alguno de ellos, ya sea en cuanto a cantidad o a calidad, ocasionará un incremento en la osmolaridad de la lágrima, siendo esta la causa de la mayoría de las alteraciones encontradas en el síndrome de ojo seco [4].

La hiperosmolaridad lagrimal causa daño al epitelio de la superficie al activar una cascada de eventos inflamatorios, con la consecuente liberación de mediadores de inflación en la lágrima. El daño epitelial implica muerte celular por apoptosis, pérdida de células caliciformes y reducción de la secreción de mucina. Esto conduce a la inestabilidad de la lágrima; a su vez, esta inestabilidad exacerba la hiperosmolaridad con la consecuente alteración de la superficie ocular, generándose así un círculo vicioso [5].

Se considera a la hiperosmolaridad lagrimal como la característica distintiva de sequedad ocular. El valor de corte recomendado es de $316 \mathrm{mOsm} / \mathrm{L}$. Por tanto, la evaluación de la osmolaridad de la lágrima en el diagnóstico de ojo seco, proporciona información valiosa sobre el equilibrio de los elementos que la componen [6].

Dada la importancia de la lágrima en la fisiología de la superficie ocular, es primordial disponer de métodos confiables que permitan medir y caracterizar su estructura, sus condiciones de estabilidad y calidad [7]. Actualmente existen diferentes pruebas que permiten estudiar la película lagrimal. En este campo, la evaluación de la osmolaridad ha sido descrita como el test "Gold Standard", esta prueba ha demostrado una precisión superior en comparación con otras, tales como los tests de Schirmer y las tinciones con Rosa de Bengala, Fluoresceína y Lisamina verde [8].

El test de Ferning o prueba de cristalización lagrimal analiza la calidad de la lágrima a partir de su nivel de osmolaridad, basándose en patrones en forma de helecho que se revelan cuando el componente acuoso de la lágrima se evapora sobre una lámina de cristal en el laboratorio. Las primeras observaciones de cristalización lagrimal fueron reportadas en 1791 por Fourcroy y Vauquelin. Sin embargo, fue Papanicolaou quien en 1946 observó este fenómeno al someter a evaporación moco cervical humano [9]. Estudios realizados in vitro han podido demostrar que los cristales se producen por la mezcla de soluciones electrolíticas con azúcares, ácidos hiaurónicos, proteínas y aminoácidos presentes en la sustancia por estudiar [10].

Rolando, en 1984, postuló que la conformación de los helechos se daba gracias a la cristalización de la porción mucinosa de la lágrima, a partir de esto, estableció una escala cualitativa para clasificar los helechos de acuerdo con su patrón [11]. Años después, a inicios de la década de los 90 Liotet y col. postularon que, en la cristalización lagrimal además de la mucina interviene la concentración de proteínas, carga iónica y la relación existente entre proteínas y cloruro de sodio. Esta teoría complementó a la expuesta años atrás por su precursor [12]. 
El procedimiento del test de Ferning consiste en extraer una muestra del fluido presente en el menisco lagrimal ocular, a través de un tubo capilar estéril; esa muestra es depositada en una lámina portaobjeto, donde debe permanecer durante al menos 10 minutos expuesta a temperatura ambiente. Luego de la evaporación de la porción líquida de la lágrima, los elementos sólidos restantes, constituidos por iones disueltos y los demás materiales ya mencionados, adoptan la forma del patrón en helecho, que se analiza a través de un microscopio óptico, el cual debe tener una capacidad mínima de entre 100x - 400x (aumentos) [12].

Se han propuesto diferentes escalas para clasificar las características de los patrones de helecho lagrimal, adoptando en general la escala de Rolando como el principal método utilizado en trabajos publicados anteriormente en esta área. Esta escala consta de siete estadíos clasificatorios. Sin embargo, esta no se desarrolló originalmente para obtener resultados reproducibles, ni constituye un instrumento de clasificación estandarizado, sus 7 patrones surgieron en forma más o menos arbitraria [9].

Ali M. Masmali y col. proponen en el 2014 la denominada Escala de Masmali. Esta se basa igualmente, en los resultados del test de Ferning; representa gráficamente los helechos lagrimales y los clasifica de acuerdo con su conformación Figura 1. Los divide en cinco estadíos o grados, según el nivel de osmolaridad hallada en la película lagrimal [9].

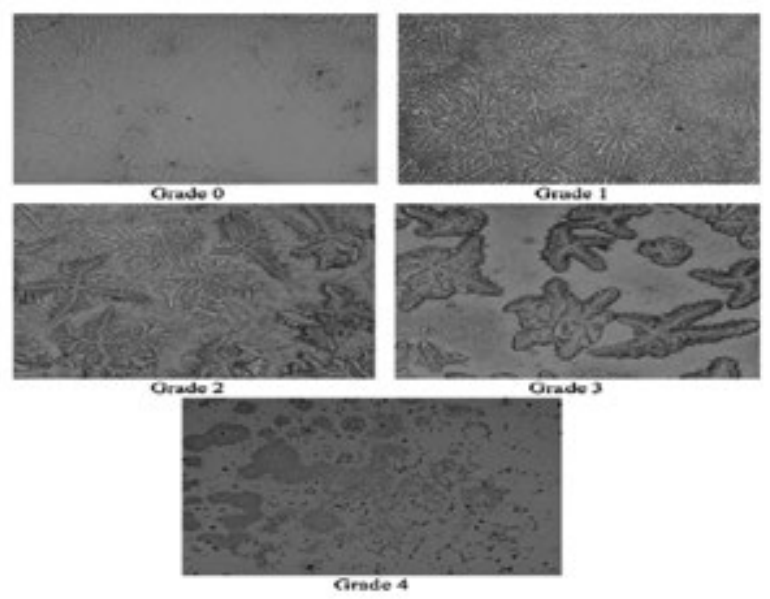

Figura 1. Escala de Masmali [9].

El grado 0 tiene el fenómeno completo del patrón de helecho, sin espacios o brechas entre los helechos y las ramas; en el grado 1, la densidad de helechos y ramas está disminuida con la aparición de pequeños espacios y huecos entre ellos. En el grado 2, los espacios y huecos aumentan, y en el grado 3 ya no existen helechos, pero sí hay presencia de grandes cristales. En el grado 4, el fenómeno del patrón de helecho está totalmente ausente [9]. A mayor densidad de los elementos sólidos de la lágrima, se afirma que el paciente se encuentra sano y con su lágrima conservada; mientras que, cuando el patrón está fragmentado, esto se asocia con un deterioro y modificación de la osmolaridad, esto depende de la concentración de electrolitos en el fluido ocular [11].

La clasificación de Masmali, es un método de valoración aceptado internacionalmente y aplicado por especialistas en segmento anterior ocular para el estudio de la osmolaridad lagrimal. La literatura científica disponible sobre la aplicación y confiabilidad de esta escala es escasa. En Colombia no hay estudios reportados que aborden esta temática.

El propósito de la presente investigación radicó en evaluar mediante observación y aplicación de pruebas estadísticas, la confiabilidad de la escala de Masmali para determinar si constituye un método recomendable en la evaluación de la osmolaridad lagrimal.

\section{MATERIALES Y MÉTODOS}

En todo momento se garantizó el manejo ético, anónimo y confidencial de la información y del nombre de quienes participaron. Esta investigación siguió lo establecido en la Resolución 008430 de 1993 del Ministerio de Salud de Colombia, por la cual se establecen las normas científicas, técnicas y administrativas para la investigación en salud [13].

La recolección y registro de la información se obtuvo mediante un formato de identificación de pacientes, consentimiento informado, formato de selección de participantes y formato de examen clínico, los cuales fueron diseñados para esta investigación.

Se hizo un estudio observacional analítico de evaluación de pruebas diagnósticas. La población estudio correspondió a un grupo de estudiantes del programa de Optometría de la Universidad Santo Tomás Seccional Bucaramanga, Santander, Colombia; en edades comprendidas entre 18 y 32 años. La elección de la 
población se hizo por conveniencia, ya que al estar concentrados todos los participantes en el mismo campus universitario, se facilitó el acceso para la toma de las pruebas. Todos los sujetos evaluados cumplieron con los criterios de elegibilidad.

El trabajo se ejecutó en 3 etapas: durante la primera, se accedió al listado de estudiantes activos del programa de Optometría; de este, fueron seleccionados 50 participantes aleatoriamente y se verificó que todos cumplieran con los siguientes criterios de elegibilidad: ser mayor de edad, no presentar alteraciones infecciosas de segmento anterior y aceptación voluntaria para participar en el estudio, manifestada a través de la firma del consentimiento informado.

En la segunda etapa, se llevó a cabo la toma de las muestras de lágrimas de los sacos conjuntivales de los participantes. De allí se extrajo una pequeña porción de este fluido mediante un tubo capilar. Esta se muestra en la Figura 2.

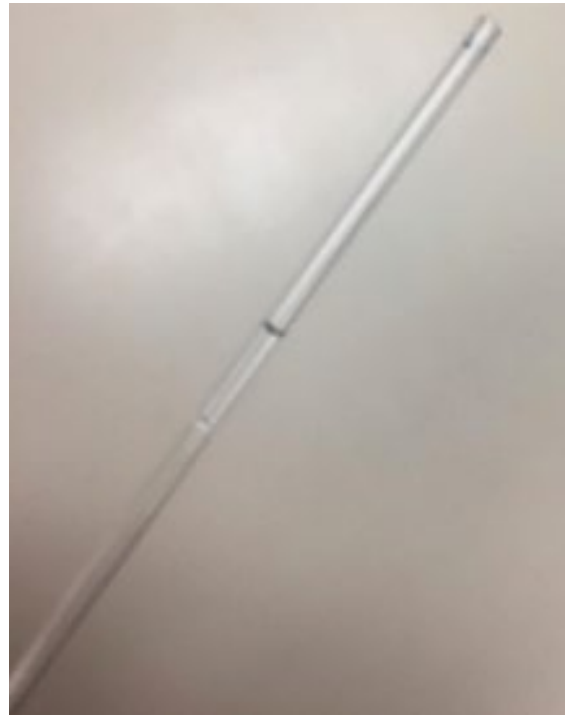

Figura 2. Muestra de lágrima en tubo capilar.

Los fluidos colectados, se sometieron a evaporación en un ambiente controlado a una temperatura constante de $20^{\circ}$ centígrados, dentro del laboratorio de histología de la institución. Posteriormente, las muestras fueron observadas a través de un estéreo microscopio.

Por último, en la tercera etapa se evaluó el patrón de cristalización. Para esto, los examinadores hicieron sus observaciones a través del microscopio en forma individual. Analizaron las imágenes obtenidas y las ubicaron según la morfología de los helechos, aplicando la escala de Masmali, para determinar el estadío osmolar de cada muestra. Figura 3.
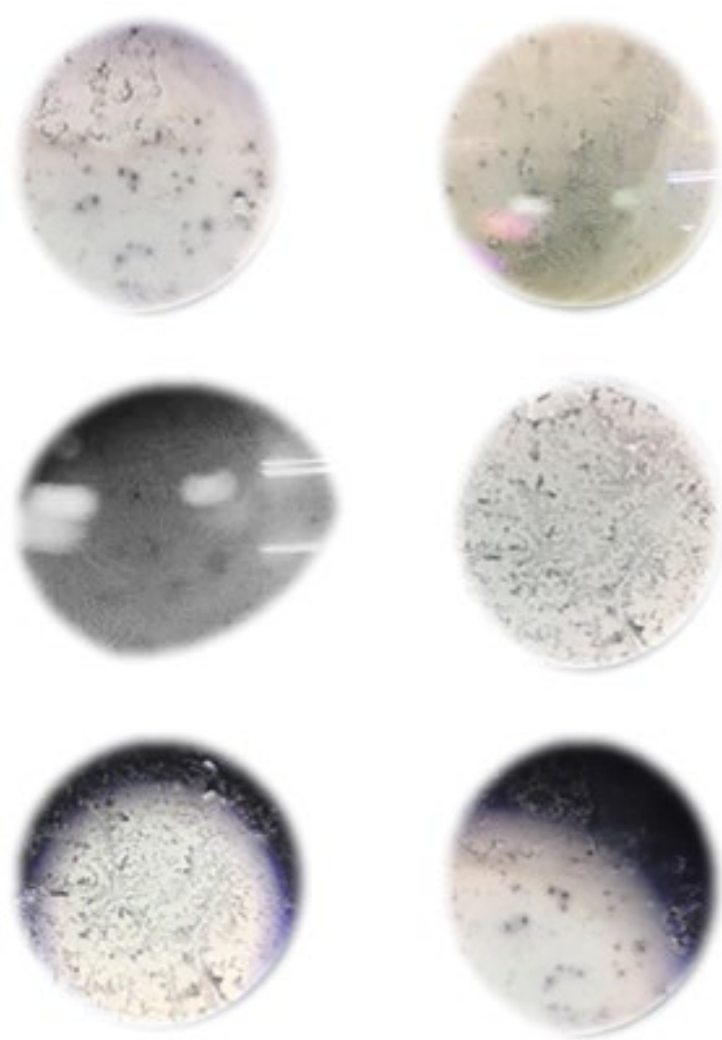

Figura 3. Muestras de patrones de helechos de lágrimas secas de los participantes, observadas a través del microscopio.

Posteriormente, se llevó a cabo el procesamiento de la información recolectada. Para este análisis se tuvieron en cuenta variables sociodemográficas y variables clínicas, a partir de los resultados obtenidos por los observadores en el test de cristalización al emplear la escala.

Se hizo un primer análisis univariado, distribución de frecuencias para las variables cualitativas y medidas de tendencia central y dispersión para las cuantitativas. En el análisis bivariado fueron evaluadas las diferencias halladas en los reportes de la escala de Masmali por los evaluadores. La reproducibilidad se evaluó con un coeficiente Kappa ponderado, por la capacidad que este 
tiene para reflejar la concordancia inter-observador [14]. Los datos se procesaron con el paquete estadístico EpiData.

Durante la toma de muestras, no se presentaron accidentes o eventos adversos relacionados con el uso de las micropipetas para la recolección de lágrima.

\section{RESULTADOS}

La población objeto estuvo conformada por 50 estudiantes en edades comprendidas entre 18 y 32 años, la edad predominante fue 19 años (36\%), con mayor presencia del género femenino $88 \%(\mathrm{n}=44)$.

En referencia a la interpretación de la escala de Masmali por parte de los investigadores, se encontró que tanto para el evaluador 1 como para el evaluador 2, el patrón más frecuente fue el 2 con $32 \%$ y $36 \%$, respectivamente. Mientras que el patrón que se presentó con menor frecuencia fue el 4 , con un $0 \%$ para el evaluador 1, y $2 \%$ para el evaluador 2 . En cuanto a la cantidad de muestras interpretadas, no se observa una diferencia estadísticamente significativa en la observación de alguno de los patrones. Sin embargo, vale la pena mencionar que en el patrón 2 se observa una mayor diferencia entre los dos evaluadores (4\%) (Tabla 1).

Los resultados de la escala de Masmali muestran que el $90 \%$ de los participantes tienen buena calidad de la capa mucinosa en ambos evaluadores.
En cuanto a la cantidad de muestras interpretadas por ambos evaluadores, no se observó una diferencia significativa de los patrones de helecho lagrimal 0,1 , 2 y 3 . En el patrón 4 se obtuvo una diferencia en la concordancia, vale mencionar que esto solo se presentó en una sola muestra. El nivel de concordancia superó el $50 \%$ de efectividad, mostrando una mejor concordancia en el patrón grado 2 de la escala estudiada, seguidos por los grados 0,1 y 3 . En la tabla 2 se presentan estos resultados. Los resultados del estudio demostraron que la concordancia inter evaluador fue moderada con un valor Kappa Ponderado de 0,5658 con un 88\% de acuerdo, un error estándar Kappa de 0,0995 y un valor p menor a 0,0001 .

Tabla 2. Porcentaje de concordancia entre evaluadores

\begin{tabular}{cc}
\hline Patrones con grado & Porcentaje \\
\hline 0 & $60,00 \%$ \\
\hline 1 & $57,14 \%$ \\
\hline 2 & $68,75 \%$ \\
\hline 3 & $60,00 \%$ \\
\hline 4 & $0,00 \%$ \\
\hline
\end{tabular}

Fuente: Autores.

\section{DISCUSIÓN}

El ojo seco es ocasionado por cualquier alteración que involucre a la unidad funcional lagrimal (UFL). A esta unidad la conforman el conjunto de todas las glándulas encargadas de la producción de los diferentes componentes de la lágrima, la superficie ocular, los pár-

Tabla 1. Interpretación de la Escala de Masmali

\begin{tabular}{ccccc}
\hline \multirow{2}{*}{$\begin{array}{c}\text { Patrones } \\
\text { con grado }\end{array}$} & \multicolumn{2}{c}{ Evaluador 1 } & \multicolumn{2}{c}{ Evaluador 2 } \\
\cline { 2 - 5 } & absocuencia & $\begin{array}{c}\text { Frecuencia } \\
\text { relativa (\%) }\end{array}$ & $\begin{array}{c}\text { Frecuencia } \\
\text { absoluta }\end{array}$ & $\begin{array}{c}\text { Frecuencia } \\
\text { relativa (\%) }\end{array}$ \\
\hline 0 & 15 & 30,00 & 14 & 28,00 \\
\hline 1 & 14 & 28,00 & 13 & 26,00 \\
\hline 2 & 16 & 32,00 & 18 & 36,00 \\
\hline 3 & 5 & 10,00 & 4 & 8,00 \\
\hline 4 & 0 & 0,00 & 1 & 2,00 \\
\hline
\end{tabular}

Fuente: Autores. 
pados y los nervios sensoriales y motores asociados. La UFL controla que la producción lagrimal responda a los cambios ambientales, endocrinos, entre otros; manteniéndose en equilibrio para suplir las necesidades de la superficie anterior del ojo. Si bien, cualquier alteración de las estructuras de la UFL pueden desencadenar ojo seco, los mecanismos centrales determinantes para esta condición se relacionan con hiperosmolaridad de la película lagrimal [5].

La severidad del ojo seco varía y debe ser diferenciada de otras alteraciones sintomáticas de la superficie con manifestaciones similares. El nivel de la sintomatología va desde una irritación ligera, hasta una molestia marcada, con dependencia de sustitutos lagrimales permanentes, resultando incluso en muchos casos, incapacitante, como por ejemplo en el Síndrome de Sjögren, en sus formas más complejas [6].

Por lo anterior, la evaluación de la película lagrimal es fundamental en el diagnóstico de la salud visual general y de las estructuras del segmento anterior ocular. Para ello, desde hace más de un siglo se vienen utilizando técnicas de medición de la función lagrimal, tales como el tiempo de rompimiento lagrimal (BUT), los tests de Schirmer y las tinciones con diferentes colorantes. Ya todas ellas han sido cuestionadas en su capacidad para estudiar la calidad de la lágrima. Es el caso del BUT y todas las que involucran aplicación de tintes, que han demostrado no tener gran validez por presentar baja reproducibilidad y por provocar alteraciones en la bioquímica lagrimal, inducidas por los componentes de los tintes diagnósticos [15].

La evaluación del volumen acuoso lagrimal se realiza principalmente con los tests de Schirmer. Aunque se han considerado como pruebas estándar para evaluar este aspecto, estas se ven limitadas por su naturaleza invasiva [16] y han sido cuestionadas, dado que en muchos casos pueden mostrar valores normales en pacientes con sintomatología relaciona$\mathrm{da}$ a alteraciones lagrimales, comprobadas incluso a través de otros métodos [15].

Por lo anterior, desde hace ya varios años se vienen desarrollando otros métodos de evaluación del fluido lagrimal menos invasivos, tales como la interferometría, y el estudio de la osmolaridad lagrimal [16]. Esta última permite acceder directamente al mecanismo desencadenante de sequedad ocular [6]. Los científicos reconocen que el análisis bioquímico de la osmolaridad es el camino por seguir, pero los pequeños volúmenes de lágrima disponibles en la superficie ocular hacen que el análisis bioquímico se complejice [9].

Desde el 2007 los informes del Drye Eye Workshop enfatizan en el papel de la osmolaridad de la lágrima dentro del ciclo del proceso del ojo seco [17]. Es por tanto, una prioridad en la exploración de las disfunciones lagrimales. Existe, sin embargo, una barrera para la aplicación rutinaria de las pruebas de osmolaridad; esta se relaciona con la necesidad de equipo especializado de laboratorio, para la observación de la cristalización lagrimal, por tanto, el número de profesionales que hacen estas pruebas es escaso.

La variación de los patrones obtenidos en la cristalización de la lágrima ha demostrado buena sensibilidad, especificidad y repetibilidad. Rolando fue el primero en desarrollar una escala de clasificación de los patrones de helechos lagrimales. Los categorizó en cuatro grados, Tipo I, II, III y IV [18]. Sin embargo, la varianza en los tipo I y II es grande, y no todos los tipos de patrones de helecho lagrimal están representados por esta escala [9].

Si la prueba de cristalización lagrimal representa una prueba fundamental en el estudio de las condiciones lagrimales, es importante tener una escala de clasificación reproducible, que permita catalogar acertadamente la osmolaridad y guíe el diagnóstico.

Masmali después de aplicar estudios de confiabilidad concluyó que la diversidad de patrones puede sintetizarse en solo 5 puntos, depuró de este modo la escala de Rolando, eliminando la superposición entre los grados. En estudios posteriores, ha sido el propio Masmali quien ha sometido su escala a varias pruebas para determinar su confiabilidad como método diagnóstico [9].

Los resultados obtenidos en este estudio concluyeron que la escala de Masmali es reproducible, fiable y precisa. La puntuación interevaluador mostró la fiabilidad del nivel y grado de concordancia en las pruebas realizadas. Esta confiabilidad también fue descrita por Dutta D y col en 2019, cuando determinaron que las pruebas realizadas mediante cristalización no arrojaron 
diferencias estadísticamente significativas aplicando la escala de Masmali [19]. Por su parte, Saud y col, el mismo año demostraron la fiabilidad e importancia de la prueba al aplicarla en el diagnóstico de la función lagrimal en pacientes con alteraciones tiroideas [20]. Así mismo, otras investigaciones han demostrado la utilidad de la prueba como método diagnóstico. Sharanjeet-Kaura y col evaluaron dos pruebas para examinar la película lagrimal, entre ellas, el test de cristalización en población asiática. Este estudio también arrojó muy buenos resultados, concluyen que puede utilizarse tanto en investigación clínica como en la exploración médica [18].

Masmali y colaboradores en un estudio posterior aplicaron la escala en pacientes diabéticos. Los resultados de esta investigación, al igual que los estudios mencionados anteriormente, mostraron que esta puede usarse como una prueba confiable para evaluar la calidad de la película lagrimal en personas con esta patología [21].

En otro estudio, llevado a cabo en el 2015 por los mismos autores, se llegó a un hallazgo de gran relevancia clínica, se pudo identificar que no existen diferencias significativas en el test de Ferning para las muestras de lágrimas recolectadas en distintos momentos del día, lo que sugiere que hay escasa variación diurna. Por lo tanto, los resultados que se obtienen con este método son confiables e independientes del momento del día en que se tome la prueba [22)].

El reconocimiento de la importancia y fiabilidad del test de cristalización lagrimal junto a la escala de evaluación de Masmali ha venido ganando terreno, no solo en la evaluación en humanos, sino que su uso se ha extendido a especies animales, constituyéndose una herramienta que viene utilizándose en el estudio de la calidad lagrimal de felinos, aves y reptiles. Varios estudios afirman obtener resultados confiables en estas especies [23].

Los resultados de la presente investigación concuerdan con los arriba mencionados, en que la escala de Masmali es un instrumento recomendable para la caracterización de la calidad lagrimal y el diagnóstico de las disfunciones lagrimales. Sin embargo, los estudios reportados en la literatura científica son escasos. En el contexto colombiano, este trabajo es el primero identificado que haya abordado esta temática, esto abre una línea de trabajo para futuras investigaciones con diferentes aplicaciones. Es importante continuar trabajando en el área, ya que las características poblacionales de los estudios que dieron soporte a los primeros hallazgos, distan de las de la población colombiana, dadas sus particularidades idiosincráticas y socio demográficas en general.

\section{REFERENCIAS}

[1] García-Resúa C, Lira M, Giráldez Mj, González-Pérez J, Yebra-Pimentel E. Estudio del tiempo de ruptura lagrimal en una población joven [Internet]. [citado 19 de mayo de 2021]. Disponible en: https://secontactologia. com/revista/revista-2005/06.htm

[2] Foulks GN. Treatment of dry eye disease by the non-ophthalmologist. Rheum Dis Clin North Am. 2008;34(4):987-1000, x. DOI: 10.1016/j.rdc.2008.08.008

[3] Kantor AP. Actualización clínica en ojo seco para el médico no oftalmólogo. Rev Med Clin Condes. 2010;21(6):88390. DOI: 10.1016/S0716-8640(10)70613-1

[4] Aguilar A. La hiperosmolaridad del film lagrimal en el ojo seco. Arq Bras Oftalmol. 2008;71(6 Supl):69-71. DOI: $10.1590 /$ S0004-27492008000700014

[5] Lemp MA, Foulks G. The Definition \& Classification of Dry Eye Disease. 2008 [citado 2021 junio 15]. Available from: https://www.tearfilm.org/pdfs/OM\%20-\%20Definition\%20\&\%20Classification.pdf

[6] Metodologías para diagnosticar y supervisar la enfermedad de ojo seco [Internet]. The Ocular Surface; 2007 [citado 2021 junio 16]. Available from: http://www.ojoseco. org/wpcontent/uploads/2012/09/metodologias-para-Diagnosticar-y-supervisar-la-enfermedad-de-ojoseco.pdf

[7] Barrera Castro JD, Rojas DA. Evaluación de la concordancia entre la prueba de Schirmer I y la prueba de Schirmer modificado de 1 minuto en estudiantes universitarios [Especialista]. Universidad Santo Tomás; 2020.

[8] Evans KSE, North RV, Purslow C. Tear ferning in contact lens wearers. Ophthalmic Physiol Opt. 2009;29(2):199204. DOI: $10.1111 / \mathrm{j} .1475-1313.2008 .00626 . x$

[9] Masmali AM, Murphy PJ, Purslow C. Development of a new grading scale for tear ferning. Contact Lens Anterior Eye J Br Contact Lens Assoc. 2014;37(3):178-84. DOI: 10.1016/j.clae.2013.09.011

[10] Mayorga MT. Diferencias en la variación del helecho lagrimal con el uso de lentes de contacto de hidrogel y de hidrogel de silicona. Cienc Tecnol Para Salud Vis Ocul. 2010;8(2):73-9. DOI: https://doi.org/10.19052/sv.816 
[11] Masmali AM, Purslow C, Murphy PJ. The tear ferning test: a simple clinical technique to evaluate the ocular tear film. Clin Exp Optom. 2014;97(5):399-406. DOI: $10.1111 /$ cxo. 12160

[12] Kogbe O, Liotet S, Tiffany JM. Factors responsible for tear ferning. Cornea. 1991;10(5):433-44. DOI: 10.1097/00003226-199109000-00013

[13] Resolución_8430_de_1993.pdf [Internet]. Disponible en: https://www.hospitalsanpedro.org/images/Comite_Investigacion/Resolucion_8430_de_1993.pdf

[14] Cerda LJ, Villarroel Del PL. Evaluación de la concordancia inter-observador en investigación pediátrica: Coeficiente de Kappa. Rev chil pediatr. [Internet]. 2008 [citado 2021 may 6] ;79(1):54-58. Disponible en: http://www.scielo.cl/scielo.php?script=sci_arttext\&pi$\mathrm{d}=S 0370-41062008000100008 \& \operatorname{lng}=e s$

[15] Durán P, León A, Márquez M, Veloza C, Grisales EV, Rivillas LC, et al. Evaluación de la película lagrimal con métodos diagnósticos invasivos vs método diagnóstico no invasivo. Investigaciones Andina [Internet]. 2006;8(12): Recuperado de: https://www.redalyc.org/ articulo.oa? $\mathrm{id}=239017506005$

[16] Hosaka E, Kawamorita T, Ogasawara Y, Nakayama N, Uozato H, Shimizu K, et al. Interferometry in the Evaluation of Precorneal Tear Film Thickness in Dry Eye. Am J Ophthalmol. 2011;151(1):18-23.e1. DOI: 10.1016/j. ajo.2010.07.019

[17] The Definition and Classification of Dry Eye Disease: Report of the Definition and Classification Subcommittee of the International Dry Eye Workshop. Ocul Surf. 2007;5(2):75-92. DOI: 10.1016/s1542-0124(12)70081-2
[18] Sharanjeet-Kaur, Ho CY, Mutalib HA, Ghazali AR. The Relationship Between Tear Ferning Patterns and Non-invasive Tear Break-up Time in Normal Asian Population. J Optom. 2016;9(3):175-81. DOI: 10.1016/j. optom.2015.10.004

[19] Dutta D, Kim J, Sarkes M, Nath S, Markoulli M. The repeatability of subjective and objective tear ferning assessment and its association with lipid layer thickness, non-invasive tear break-up time and comfort. Contact Lens Anterior Eye. 2019;42(4):420-7. DOI: 10.1016/j. clae.2019.04.003

[20] Alanazi SA, Alomran AA, Abusharha A, Fagehi R, Al-Johani N, El-Hiti GA, et al. An assessment of the ocular tear film in patients with thyroid disorders. Clin Ophthalmol. 2019;13:1019-26. DOI: 10.2147/OPTH.S210044

[21] Masmali AM, Maeni YA, El-Hiti GA, Murphy PJ, Almubrad T. Investigation of Ocular Tear Ferning in Controlled and Uncontrolled Diabetic Subjects. Eye Contact Lens. 2018;44 Suppl 2:S70-5. DOI: 10.1097 / ICL.0000000000000419

[22] Masmali AM, Al-Bahlal JM, El-Hiti GA, Akhtar S, Purslow C, Murphy PJ, et al. Repeatability and Diurnal Variation of Tear Ferning Test. Eye Contact Lens. septiembre de 2015;41(5):262-7. DOI: 10.1097/ ICL.0000000000000116

[23] Oriá AP, Lacerda A de J, Raposo ACS, Araújo NLLC, Portela R, Mendonça MA, et al. Comparison of Electrolyte Composition and Crystallization Patterns in Bird and Reptile Tears. Front Vet Sci. 2020;7:574. DOI: 10.3389/ fvets.2020.00574

\section{Correo de autores}

Camilo Luis Daza Martínez: camilo.daza01@ustabuca.edu.co Rafael Humberto Sánchez Fragozo: rafaelhsanchez@hotmail.com Martha Lucila Márquez García: martha.marquez@ustabuca.edu.co Juan José Barrios Arlante: juan.barrios01@ustabuca.edu.co 